\title{
RECURRENCE RELATIONS FOR THE MIDPOINT METHOD
}

\author{
J. A. EZQUERRO. M. A. HERNÁNDEZ AND M. A. SALANOVA
}

\begin{abstract}
In this paper, we present a new convergence analysis and error estimates for the Midpoint method in Banach spaces by using Newton-Kantorovich-type assumptions and a technique based on a new system of recurrence relations. Finally, we give three examples where we improve the error bounds are better given by other authors.
\end{abstract}

\section{Introduction}

Many scientific problems are like solving a nonlinear equation of the form $F(x)=0$. This equations can represent differential equations, integral equations or a system of equations in the simplest case. In order to set out these equation types in a general way, we consider $F$ as a nonlinear operator defined on a subset $\Omega$ of a Banach space $X$ with values in another Banach space $Y$. The Newton method is the most used iteration to solve those equations as a consequence of computational efficiency even less speed of convergence can be got.

Our goals in this paper is to increase the speed of convergence of Newton's method and not to increase its operational cost very much. Taking into account these goals, we consider a multipoint Newton-type method of order three called the Midpoint method studied by other authors $([3],[7])$. This method is defined for all $n \geq 0$ by

$$
\begin{aligned}
y_{n} & =x_{n}-\Gamma_{n} F\left(x_{n}\right), \quad z_{n}=x_{n}+\frac{1}{2}\left(y_{n}-x_{n}\right), \\
x_{n+1} & =x_{n}-\bar{\Gamma}_{n} F\left(x_{n}\right)
\end{aligned}
$$

where $\Gamma_{n}=F^{\prime}\left(x_{n}\right)^{-1}$ and $\bar{\Gamma}_{n}=F^{\prime}\left(z_{n}\right)^{-1}$.

We introduce a new technique to study the convergence of (1) based on the construction of a system of recurrence relations that consists of two sequences of positive real numbers that guarantee the convergence of sequence (1) in Banach spaces. We also provide some a priori error bounds. By this new technique better convergence domains

Received February 4, 1999.

1991 Mathematics Subject Classification. 47H17, 65J15.

Key words and phrases. Nonlinear equations in Banach spaces, third-order method, recurrence relations, multipoint iteration.

Supported in part by the University of La Rioja (grants API-97/A30 and API-99/B14) and DGES(grants PB96-0120-C03-02 and PB98-0198). 
and better error bounds are obtained than the ones obtained by other authors, as we can see in the applications included at the end of the paper.

\section{Recurrence Relations}

Let $X, Y$ be Banach spaces and $F: \Omega \subseteq X \rightarrow Y$ be a nonlinear twice Fréchet differentiable operator in an open convex domain $\Omega_{0} \subseteq \Omega$. The Midpoint method to solve the equation $F(x)=0$ given by (1) can be written in the following form:

$$
\begin{aligned}
H\left(x_{n}, z_{n}\right) & =\bar{\Gamma}_{n}\left[F^{\prime}\left(z_{n}\right)-F^{\prime}\left(x_{n}\right)\right] \\
x_{n+1} & =y_{n}-H\left(x_{n}, z_{n}\right)\left(y_{n}-x_{n}\right),
\end{aligned}
$$

Let us assume that $\Gamma_{0}=F^{\prime}\left(x_{0}\right)^{-1} \in \mathcal{L}(Y, X)$ exists at some $x_{0} \in \Omega_{0}$, where $\mathcal{L}(Y, X)$ is the set of bounded linear operators from $Y$ into $X$.

Throughout this paper we assume that

(i) $\left\|\Gamma_{0}\right\| \leq \beta$.

(ii) $\left\|\Gamma_{0} F\left(X_{0}\right)\right\| \leq \eta$.

(iii) $\left\|F^{\prime \prime}(x)\right\| \leq M, x \in \Omega_{0}$.

(iv) $\left\|F^{\prime \prime}(x)-F^{\prime \prime}(y)\right\| \leq K\|x-y\|, x, y \in \Omega_{0}$.

Let us denote

$$
a_{0}=M \beta \eta, \quad b_{0}=K \beta \eta^{2} .
$$

Next we define the sequences

$$
a_{n+1}=a_{n} f\left(a_{n}\right)^{2} g\left(a_{n}, b_{n}\right), \quad b_{n+1}=b_{n} f\left(a_{n}\right)^{3} g\left(a_{n}, b_{n}\right)^{2} .
$$

where

$$
f(x)=\frac{2-x}{2-3 x}
$$

and

$$
g(x, y)=\frac{x^{2}}{(2-x)^{2}}+\frac{7 y}{24}
$$

Assuming now that $a_{0}<\frac{2}{3}$ and $y_{0} \in \Omega_{0}$ we deduce that $z_{0} \in \Omega_{0}$. Then by the initial hypotheses (i)-(iv), we have

$$
\left\|I-\Gamma_{0} F^{\prime}\left(z_{0}\right)\right\| \leq\left\|\Gamma_{0}\right\|\left\|F^{\prime}\left(x_{0}\right)-F^{\prime}\left(z_{0}\right)\right\| \leq \frac{M}{2}\left\|\Gamma_{0}\right\|\left\|y_{0}-x_{0}\right\| \leq \frac{a_{0}}{2}<1
$$

and, by the Banach lemma, $\bar{\Gamma}_{0}$ exists and $\left\|\bar{\Gamma}_{0}\right\| \leq \frac{2}{2-a_{0}} \Gamma_{0}$. On the other hand

$$
\begin{gathered}
M\left\|\Gamma_{0}\right\|\left\|y_{0}-x_{0}\right\| \leq a_{0} \\
K\left\|\Gamma_{0}\right\|\left\|y_{0}-x_{0}\right\|^{2} \leq b_{0} . \\
\left\|H\left(x_{0}, z_{0}\right)\right\|=\left\|\bar{\Gamma}_{0}\left[F^{\prime}\left(z_{0}\right)-F^{\prime}\left(x_{0}\right)\right]\right\| \leq \frac{a_{0}}{2-a_{0}} .
\end{gathered}
$$


Then $x_{1}$ is well defined and

$$
\left\|x_{1}-x_{0}\right\| \leq\left\|\bar{\Gamma}_{0} F\left(x_{0}\right)\right\| \leq\left\|\bar{\Gamma}_{0} F^{\prime}\left(x_{0}\right)\right\|\left\|\Gamma_{0} F\left(x_{0}\right)\right\| \leq \frac{2}{2-a_{0}}\left\|\Gamma_{0} F\left(x_{0}\right)\right\| .
$$

In that situation we prove the following estimates for all $n \geq 1$ :

$\left(I_{n}\right)\left\|\Gamma_{n}\right\| \leq f\left(a_{n-1}\right)\left\|\Gamma_{n-1}\right\|$,

$\left(I I_{n}\right)\left\|y_{n}-x_{n}\right\|=\left\|\Gamma_{n} F\left(x_{n}\right)\right\| \leq f\left(a_{n-1}\right) g\left(a_{n-1}, b_{n-1}\right)\left\|y_{n-1}-x_{n-1}\right\|$,

$\left(I I I_{n}\right) M\left\|\Gamma_{n}\right\|\left\|y_{n}-x_{n}\right\| \leq a_{n}$,

$\left(I V_{n}\right) K\left\|\Gamma_{n}\right\|\left\|y_{n}-x_{n}\right\|^{2} \leq b_{n}$,

$\left(V_{n}\right)\left\|\bar{\Gamma}_{n}\right\| \leq \frac{2}{2-a_{n}}\left\|\Gamma_{n}\right\|$

$\left(V I_{n}\right)\left\|H\left(x_{n}, z_{n}\right)\right\| \leq \frac{a_{n}}{2-a_{n}}$

$\left(V I I_{n}\right)\left\|x_{n+1}-x_{n}\right\| \leq \frac{2}{2-a_{n}}\left\|\Gamma_{n} F\left(x_{n}\right)\right\|=\frac{2}{2-a_{n}}\left\|y_{n}-x_{n}\right\|$.

We proceed by mathematical induction on $n$.

$\left[\mathbf{I}_{1}\right]$ : Taking into account that $a_{0}<\frac{2}{3}$ and $x_{1} \in \Omega_{0}$, then

$$
\left\|I-\Gamma_{0} F^{\prime}\left(x_{1}\right)\right\| \leq\left\|\Gamma_{0}\right\|\left\|F^{\prime}\left(x_{0}\right)-F^{\prime}\left(x_{1}\right)\right\| \leq M\left\|\Gamma_{0}\right\|\left\|x_{1}-x_{0}\right\| \leq \frac{2 a_{0}}{2-a_{0}}<1
$$

and, by the Banach lemma, $\Gamma_{1}$ exists and

$$
\left\|\Gamma_{1}\right\| \leq \frac{\left\|\Gamma_{0}\right\|}{1-\left\|I-\Gamma_{0} F^{\prime}\left(x_{1}\right)\right\|} \leq f\left(a_{0}\right)\left\|\Gamma_{0}\right\|
$$

Hence $\left\|\Gamma_{1} F^{\prime}\left(x_{0}\right)\right\| \leq f\left(a_{0}\right)$.

$\left[\mathbf{I I}_{1}\right]$ : Using Taylor's formula, if $y_{0} \in \Omega_{0}$, then $z_{0} \in \Omega_{0}$ and we have

$$
\begin{aligned}
F\left(x_{1}\right)= & F\left(y_{0}\right)+F^{\prime}\left(y_{0}\right)\left(x_{1}-y_{0}\right)+\int_{y_{0}}^{x_{1}} F^{\prime \prime}(x)\left(x_{1}-x\right) d x \\
= & \int_{0}^{1} F^{\prime \prime}\left(y_{0}+t\left(x_{1}-y_{0}\right)\right)(1-t) d t\left(x_{1}-y_{0}\right)^{2} \\
& -\int_{0}^{1} F^{\prime \prime}\left(z_{0}+t\left(y_{0}-z_{0}\right)\right)\left(y_{0}-z_{0}\right) d t H\left(x_{0}, z_{0}\right)\left(y_{0}-x_{0}\right) \\
& +\int_{0}^{1}\left[F^{\prime \prime}\left(x_{0}+t\left(y_{0}-x_{0}\right)\right)-F^{\prime \prime}\left(x_{0}\right)\right](1-t) d t\left(y_{0}-x_{0}\right)^{2} \\
& +\frac{1}{2} \int_{0}^{1}\left[F^{\prime \prime}\left(x_{0}\right)-F^{\prime \prime}\left(x_{0}+\frac{1}{2} t\left(y_{0}-x_{0}\right)\right)\right] d t\left(y_{0}-x_{0}\right)^{2} .
\end{aligned}
$$

So

$$
\left\|y_{1}-x_{1}\right\|=\left\|\Gamma_{1} F\left(x_{1}\right)\right\| \leq f\left(a_{0}\right) g\left(a_{0}, b_{0}\right)\left\|y_{0}-x_{0}\right\| .
$$

$\left[\mathbf{I I I}_{1}\right]:$

$$
M\left\|\Gamma_{1}\right\|\left\|y_{1}-x_{1}\right\| \leq M\left\|\Gamma_{0}\right\|\left\|y_{0}-x_{0}\right\| f\left(a_{0}\right)^{2} g\left(a_{0}, b_{0}\right) \leq a_{1} .
$$

$\left[\mathbf{I V}_{1}\right]:$

$$
K\left\|\Gamma_{1}\right\|\left\|y_{1}-x_{1}\right\|^{2} \leq K\left\|\Gamma_{0}\right\|\left\|y_{0}-x_{0}\right\|^{2} f\left(a_{0}\right)^{3} g\left(a_{0}, b_{0}\right)^{2} \leq b_{1}
$$


$\left[\mathbf{V}_{1}\right]$ : Notice that if $a_{1}<\frac{2}{3}$ and $y_{1} \in \Omega_{0}$, then $z_{1} \in \Omega_{0}$ and

$$
\left\|I-\Gamma_{1} F^{\prime}\left(z_{1}\right)\right\| \leq\left\|\Gamma_{1}\right\|\left\|F^{\prime}\left(x_{1}\right)-F^{\prime}\left(z_{1}\right)\right\|<\frac{M}{2}\left\|\Gamma_{1}\right\|\left\|y_{1}-x_{1}\right\| \leq \frac{a_{1}}{2} \leq 1 .
$$

and, by the Banach lemma. $\bar{\Gamma}_{1}$ exists and $\left\|\bar{\Gamma}_{1}\right\| \leq \frac{2}{2-a_{1}}\left\|\Gamma_{1}\right\|$. Consequently, $\left\|\bar{\Gamma}_{1} F^{\prime}\left(x_{1}\right)\right\| \leq$ $\frac{2}{2-a_{1}}$.

$\left[\mathbf{V I}_{1}\right]$ :

$$
\left\|H\left(x_{1}, z_{1}\right)\right\|=\| \bar{\Gamma}_{1}\left[F^{\prime}\left(z_{1}\right)-F^{\prime}\left(x_{1}\right) \| \leq \frac{a_{1}}{2-a_{1}} .\right.
$$

$\left[\mathrm{VII}_{1}\right]$ :

$$
\left\|x_{2}-x_{1}\right\|=\left\|\bar{\Gamma}_{1} F\left(x_{1}\right)\right\| \leq\left\|\bar{\Gamma}_{1} F^{\prime}\left(x_{1}\right)\right\|\left\|\Gamma_{1} F\left(x_{1}\right)\right\| \leq \frac{2}{2-a_{1}}\left\|y_{1}-x_{1}\right\| .
$$

Following an inductive procedure and assuming for all $n \geq 0$ that $x_{n+1}, y_{n} \in \Omega_{0}$ and $a_{n}<\frac{2}{3}$, items $\left[\mathbf{I}_{n}\right]-\left[\mathbf{V I I} \mathbf{I}_{n}\right]$ are true for a fixed $n \geq 1$. We can prove $\left[\mathbf{I}_{n+1}\right]$-[ $\left[\mathbf{V}_{n+1}\right]$ in the a similar way and the induction is complete.

To study the sequence $\left\{x_{n}\right\}$ defined in a Banach space we analyse the real sequences $\left\{a_{n}\right\}$ and $\left\{b_{n}\right\}$. To establish the convergence of $\left\{x_{n}\right\}$ we only have to prove that $\left\{x_{n}\right\}$ is a Cauchy sequence and the following assumptions:

$$
x_{n+1}, y_{n} \in \Omega_{0} \text { and } a_{n}<\frac{2}{3}, \quad n \in \mathbb{N} .
$$

All of that is the aim of the following section.

\section{A Convergence Study}

In this section, we study the sequences $\left\{a_{n}\right\}$ and $\left\{b_{n}\right\}$ defined in (4) to prove the convergence of (1). First at all. We give a technical lemma, whose proof is trivial, that includes the results over the functions $f(x)$ and $g(x, y)$ given respectively by (5) and (6).

Lemma 1. Let $f$ and $g$ two real functions give in (5) and (6) respectively. Then

(i) $f$ is increasing and $f(x)>1$ for $x \in(0,2 / 3)$,

(ii) $g$ is increasing for all $x \in(0,2 / 3)$ and for all $y>0$.

(iii) $f(\gamma x)<f(x)$ and $g\left(\gamma x, \gamma^{2} y\right)<\gamma^{2} g(x, y)$ for $\gamma \in(0,1), y>0$ and $x \in(0,2 / 3)$.

Some properties for the sequences $\left\{a_{n}\right\}$ and $\left\{b_{n}\right\}$ given by (4) are now provided. From now on, let us denote

$$
h(x)=\frac{96(1-x)(1-2 x)}{7(2-x)^{2}}
$$

Lemma 2. Let $f$ and $g$ be as before, $a_{0} \in(0,1 / 2)$ and $b_{0}<h\left(a_{0}\right)$. Then

(i) $f\left(a_{0}\right)^{2} g\left(a_{0}, b_{0}\right)<1$,

(ii) the sequences $\left\{a_{n}\right\}$ and $\left\{b_{n}\right\}$ are decreasing 
(iii) $a_{n}<2 / 3$ for all $n \geq 0$.

Proof. From the hypotheses, (i) follows immediately. We show (ii) by mathematical induction on $n$. The facts that $0<a_{1}<a_{0}$ and $0<b_{1}<b_{0}$ follow by previous (i) and lemma 1 (i). Next, it is supposed that $a_{j}<a_{j-1}$ and $0<b_{j}<b_{j-1}$ for $j=1,2, \ldots, n$. Then

$$
a_{n+1}=a_{n} f\left(a_{n}\right)^{2} g\left(a_{n}, b_{n}\right)<a_{n-1} f\left(a_{n-1}\right)^{2} g\left(a_{n-1}, b_{n-1}\right)=a_{n}
$$

since $f$ and $g$ are increasing and $f(x)>1$ for $x \in(0,2 / 3)$.

By a similar reasoning, we have

$$
\begin{aligned}
b_{n+1} & =b_{n} f\left(a_{n}\right)^{3} g\left(a_{n}, b_{n}\right)^{2}<b_{n} f\left(a_{n}\right)^{4} g\left(a_{n}, b_{n}\right)^{2}<b_{n}\left[f\left(a_{n}\right)^{2} g\left(a_{n}, b_{n}\right)\right]^{2} \\
& <b_{n}\left[f\left(a_{0}\right)^{2} g\left(a_{0}, b_{0}\right)\right]^{2}<b_{n}
\end{aligned}
$$

Finally, for all $n \geq 0$, we have $a_{n}<2 / 3$ since $\left\{a_{n}\right\}$ is a decreasing sequence and $a_{0} \in$ $(0,1 / 2)$. The proof is complete.

Lemma 3. Let us suppose the hypotheses of lemma 2 and define $\gamma=a_{1} / a_{0}$. Then, we have

(i $\left.i_{n}\right) a_{n}<\gamma^{3^{n-1}} a_{n-1}<\gamma^{\frac{3^{n}-1}{2}} a_{0}$ and $b_{n}<\left(\gamma^{3^{n-1}}\right)^{2} b_{n-1}<\gamma^{3^{n}-1} b_{0}$, for $n \geq 2$.

(ii $\left.{ }_{n}\right) f\left(a_{n}\right) g\left(a_{n}, b_{n}\right)<\gamma^{3^{n}} \frac{f\left(a_{0}\right) g\left(a_{0}, b_{0}\right)}{\gamma}=\frac{\gamma^{3^{n}}}{f\left(a_{0}\right)}$, for $n \geq 1$.

Proof. We prove $\left(\mathbf{i}_{n}\right)$ following an inductive procedure. Taking into account that $a_{1}=\gamma a_{0}$ and

$$
b_{1}=b_{0} f\left(a_{0}\right)^{3} g\left(a_{0}, b_{0}\right)^{2}<b_{0}\left[f\left(a_{0}\right)^{2} g\left(a_{0}, b_{0}\right)\right]^{2}=\gamma^{2} b_{0} .
$$

$\left(\mathrm{i}_{2}\right)$ holds by lemma 1 (iii). If we suppose that $\left(\mathbf{i}_{n}\right)$ is true, then

$$
\begin{aligned}
a_{n+1} & =a_{n} f\left(a_{n}\right)^{2} g\left(a_{n}, b_{n}\right)<\gamma^{3^{n-1}} a_{n-1} f\left(\gamma^{3 n-1} a_{n-1}\right)^{2} g\left(\gamma^{3^{n-1}} a_{n-1},\left(\gamma^{3^{n-1}}\right)^{2} b_{n-1}\right) \\
& <\gamma^{3^{n-1}}\left(\gamma^{3^{n-1}}\right)^{2} a_{n-1} f\left(a_{n-1}\right)^{2} g\left(a_{n-1}, b_{n-1}\right)=\gamma^{3^{n}} a_{n} .
\end{aligned}
$$

Similarly,

$$
b_{n+1}=b_{n} f\left(a_{n}\right)^{3} g\left(a_{n}, b_{n}\right)^{2}<b_{n} f\left(a_{n}\right)^{4} g\left(a_{n}, b_{n}\right)^{2}<b_{n}\left(\frac{a_{n+1}}{a_{n}}\right)^{2}<\left(\gamma^{3^{n}}\right)^{2} b_{n} .
$$

Moreover,

$$
\begin{aligned}
& a_{n+1}<\gamma^{3^{n}} a_{n}<\gamma^{3 n} \gamma^{\frac{3^{n}-1}{2}} a_{0}=\gamma^{\frac{3^{n+1}-1}{2}} a_{0}, \\
& b_{n+1}<\left(\gamma^{3^{n}}\right)^{2} b_{n}<\left(\gamma^{3^{n}}\right)^{2} \gamma^{3^{n}-1} b_{0}=\gamma^{3^{n+1}-1} b_{0} .
\end{aligned}
$$

On the other hand, we observe that

$$
f\left(a_{n}\right) g\left(a_{n} b_{n}\right)<f\left(\gamma^{\frac{3^{n}-1}{2}} a_{0}\right) g\left(\gamma^{\frac{3^{n}-1}{2}} a_{0}, \gamma^{3^{n}-1} b_{0}\right)<\gamma^{3^{n}-1} f\left(a_{0}\right) g\left(a_{0}, b_{0}\right)=\frac{\gamma^{3^{n}}}{f\left(a_{0}\right)}
$$


The proof is complete.

So we are ready to state the following result on the convergence of iteration (1).

Theorem 1. Let $X, Y$ be Banach spaces and $F: \Omega \subseteq X \rightarrow Y$ be a nonlinear twice Fréchet differentiable operator in an open convex domain $\Omega_{0} \subseteq \Omega$. Let us assume that $\Gamma_{0}=F^{\prime}\left(x_{0}\right)^{-1} \in \mathcal{L}(Y, X)$ exists at some $x_{0} \in \Omega_{0}$ and assumptions $(i)-(i v)$ are satisfied. Let us denote $a_{0}=M \beta \eta$ and $b_{0}=K \beta \eta^{2}$. Suppose that $0<a_{0}<1 / 2$ and $b_{0}<h\left(a_{0}\right)$, where $h(x)$ is the function defined in (7). Then, if $\overline{B\left(x_{0}, R \eta\right)}=\left\{x \in X ;\left\|x-x_{0}\right\| \leq\right.$ $R \eta\} \subseteq \Omega_{0}$, where $R=\frac{2}{2-a_{0}} \frac{1}{1-\Delta}$ with $\Delta=\frac{1}{f\left(a_{0}\right)}$, the sequence $\left\{x_{n}\right\}$ defined in (1) and starting at $x_{0}$ converges at least $R$-cubically to a solution $x^{*}$ of the equation $F(x)=0$. In that case, the solution $x^{*}$ and the iterates $x_{n}, y_{n}, z_{n}$ belong to $\overline{B\left(x_{0}, R \eta\right)}$, and $x^{*}$ is the only solution of $F(x)=0$ in $B\left(x_{0}, \frac{2}{M \beta}-R \eta\right) \cap \Omega_{0}$.

Furthermore, the following error estimates holds:

$$
\left\|x^{*}-x_{n+1}\right\| \leq \frac{2}{2-a_{0}}\left(\gamma^{\frac{3^{n}-1}{2}}\right) \frac{\Delta^{n}}{1-\Delta} \eta .
$$

Proof. Firstly, we prove that $\left\{x_{n}\right\}$ is a Cauchy sequence. Observe that

$$
\begin{aligned}
\left\|x_{n+1}-x_{n}\right\| & \leq \frac{2}{2-a_{n}}\left\|y_{n}-x_{n}\right\| \leq \frac{2}{2-a_{0}} f\left(a_{n-1}\right) g\left(a_{n-1}, b_{n-1}\right)\left\|y_{n-1}-x_{n-1}\right\| \\
& \leq \cdots \leq \frac{2}{2-a_{0}}\left\|y_{0}-x_{0}\right\| \prod_{j=0}^{n-1} f\left(a_{j}\right) g\left(a_{j}, b_{j}\right)
\end{aligned}
$$

as a consequence of estimate $\left[\mathbf{I I}_{n}\right]$. Then, from lemma 3 , it follows that

$$
\prod_{j=0}^{n-1} f\left(a_{j}\right) g\left(a_{j}, b_{j}\right) \leq \prod_{j=0}^{n-1}\left(\gamma^{3 j} \Delta\right)=\gamma^{\frac{3^{n}-1}{2}} \Delta^{n}
$$

where $\gamma=a_{1} / a_{0}<1$ and $\Delta=\frac{1}{f\left(a_{0}\right)}<1$.

Then

$$
\begin{aligned}
\left\|x_{n+m}-x_{n}\right\| & \leq\left\|x_{n+m}-x_{n+m-1}\right\|+\left\|x_{n+m-1}-x_{n+m-2}\right\|+\cdots+\left\|x_{n+1}-x_{n}\right\| \\
& \leq \frac{2 \eta}{2-a_{n+m-1}} \prod_{j=0}^{n+m-2} f\left(a_{j}\right) g\left(a_{j}, b_{j}\right)+\cdots+\frac{2 \eta}{2-a_{n}} \prod_{j=0}^{n-1} f\left(a_{j}\right) g\left(a_{j}, b_{j}\right) \\
& \leq \frac{2 \eta}{2-a_{n}}\left(\gamma^{\frac{2^{n+m-1}-1}{2}} \Delta^{n+m-1}+\cdots+\gamma^{\frac{3^{n}-1}{2}} \Delta^{n}\right) \\
& \leq \frac{\left(\gamma^{\frac{3 n-1}{2}}\right) 2 \eta}{2-a_{0}} \frac{\Delta^{n}\left(1-\Delta^{m}\right)}{1-\Delta} .
\end{aligned}
$$


and, for $n=0$, we obtain

$$
\left\|x_{m}-x_{0}\right\| \leq \frac{2 \eta}{2-a_{0}} \frac{\left(1-\Delta^{m}\right)}{1-\Delta}<R \eta .
$$

By letting $m \rightarrow \infty$ in (9), we get (8). Similarly, we have $y_{n}, z_{n} \in \overline{B\left(x_{0}, R \eta\right)}$ for all $n \geq 0$.

To see that $x^{*}$ is a solution of $F(x)=0$, we have $\left\|\Gamma_{n} F\left(x_{n}\right)\right\| \rightarrow 0$ as $n \rightarrow \infty$. Taking into account that $\left\|F\left(x_{n}\right)\right\| \leq\left\|F^{\prime}\left(x_{n}\right)\right\|\left\|\Gamma_{n} F\left(x_{n}\right)\right\|$ and the sequence $\left\{\left\|F^{\prime}\left(x_{n}\right)\right\|\right\}$ is bounded, we infer that $\left\|F\left(x_{n}\right)\right\| \rightarrow 0$ as $n \rightarrow \infty$. Consequently, we obtain $F\left(x^{*}\right)=0$ by the continuity of $F$.

To prove the uniqueness, we assume a some other solution $y^{*}$ of $F(x)=0$ in $B\left(x_{0}, \frac{2}{M \beta}-R \eta\right) \cap \Omega_{0}$. From the approximation

$$
0=F\left(y^{*}\right)-F\left(x^{*}\right)=\int_{0}^{1} F^{\prime}\left(x^{*}+t\left(y^{*}-x^{*}\right)\right) d t\left(y^{*}-x^{*}\right) .
$$

we have to prove that the operator $\int_{0}^{1} F^{\prime}\left(x^{*}=t\left(y^{*}-x^{*}\right)\right) d t$ is invertible and then $y^{*}=x^{*}$. Indeed, from

$$
\begin{gathered}
\quad\left\|\Gamma_{0}\right\| \int_{0}^{1}\left\|F^{\prime}\left(x^{*}+t\left(y^{*}-x^{*}\right)\right)-F^{\prime}\left(x_{0}\right)\right\| d t \leq K \beta \int_{0}^{1}\left\|x^{*}+t\left(y^{*}-x^{*}\right)-x_{0}\right\| d t \\
\leq K \beta \int_{0}^{1}\left((1-t)\left\|x^{*}-x_{0}\right\|+t\left\|y^{*}-x_{0}\right\|\right) d t<\frac{K \beta}{2}\left(R \eta+\frac{2}{K \beta}-R \eta\right)=1,
\end{gathered}
$$

it follows that $\left[\int_{0}^{1} F^{\prime}\left(x^{*}+t\left(y^{*}-x^{*}\right)\right) d t\right]^{-1}$ exists.

Finally, we deduce that the $R$-order of convergence [5] of sequence (3) is at least three. This conclusion follows by (8), since that

$$
\left\|x^{*}-x_{n}\right\|<\frac{2 \eta}{\left(2-a_{0}\right) \gamma(1-\Delta)} \gamma^{3^{n}}, \quad n \geq 0 .
$$

The proof is complete.

\section{Applications}

Finally we give three examples to illustrate the previous results. We take three functions used as a test in several papers. In these examples, we compare the convergence domains and the the error bounds obtained for other authors for his method.

Example 1. ([2]) Let us consider $F(x)=x^{3}-10, x_{0}=2$ and denote $x^{*}$ the positive root of $F(x)=0$. We will give an upper bound $C$ for $10^{7}\left\|x^{*}-x_{2}\right\|$, where $x_{2}$ is the second iterate of the Midpoint method. Starting at the interval $[1,3]$ we have $\beta=1 / 12$, $\eta=1 / 6, M=18, K=6$, and consecuently, $a_{0}=1 / 4$ and $b_{0}=1 / 72$. Taking into account that our optimal estimates are respect to consecutive points. We consider

$$
\left\|x^{*}-x_{2}\right\| \leq\left\|x^{*}-x_{4}\right\|+\left\|x_{4}-x_{3}\right\|+\left\|x_{3}-x_{2}\right\|
$$


to get $C=2.48367$. For the same function the upper bound $C$ that we obtain from ([3]) by method (1) is 5.00483 .

Example 2. ([4]) Let us consider $F: C[0,1] \rightarrow C[0,1]$ the operator defined by

$$
F(x)(s)=x(s)-s+\frac{1}{2} \int_{0}^{1} s \cos (x(t)) d t .
$$

where $C[0,1]$ is the space of all continuous functions defined on the interval $[0,1]$ with the sup norm $\|\cdot\|=\|\cdot\|_{\infty}$.

If we take $x_{0}=x_{0}(s)=s$ as a starting point, we obtain the upper bound $C=$ 0.000156396 for $\left\|x^{*}-x_{2}\right\|$, where $x_{2}$ is the second iterate of the Midpoint method. The hypotheses given by Dong Chen and Argyors in ([3]) are not satisfied and their convergence analysis cannot then be applied to the above integral equation.

Example 3. ([1]) Let $X=C[0,1]$ be the space of continuous functions defined on the interval $[0,1]$, with the max-norm and consider the integral equation $F(x)=0$, where

$$
F(x)(s)=\lambda x(s) \int_{0}^{1} \frac{s}{s+t} x(t) d t-x(s)+1 .
$$

with $s \in[0,1], x \in C[0,1]$ and $0<\lambda \leq 2$. Integral equations of this kind (called Chandrasekhar equations) arise in elasticity or neutron transport problems (see [1], [6]).

For $\lambda=1 / 4$, and starting at $x_{0}=x_{0}(s)=1$, we obtain (see [1]), $\left\|\Gamma_{0}\right\|=1.5309421=$ $\beta$. $\left\|\Gamma_{0} F\left(x_{0}\right)\right\| \leq 0.2651971=\eta,\left\|F^{\prime \prime}(x)\right\| \leq 0.3465735=M$ and $K=0$. So $a_{0}=M \beta \eta=$ 0.140659 and $b_{0}=0$. We give the upper bound $C=5.59621$ to the number $10^{10}\left\|x^{*}-x_{2}\right\|$, where $x_{2}$ is the second iterate of the Midpoint method. Taking into account the error estimates given in ([3]), the upper bound for $10^{10}\left\|x^{*}-x_{2}\right\|$ is $C=5.67272$. In this case, the improvement brought about by our technique is a little significant.

\section{References}

[1] I. K. Argyros, On a class of nonlinear integral equations arising in neutron transport, Aequationes Mathematicae, 36(1988), 99-11.

[2] V. Candela and A. Marquina, Recurrence relations for rational cubic methods II: the Chebyshev method, Computing, 45(1990), 355-367.

[3] Dong Chen and I. K. Argyors, The midpoint method for solving nonlinear operator equations in Banach spaces, Appl. Math. Lett., 5(1992), 7-9.

[4] B. Döring, Einige Sätze über das verfahren der tangierenden hyperbeln in Banach-Räumen, Aplikace Mat., 15(1970), 418-464.

[5] F. A. Potra and V. Pták, Nondiscrete Induction and Iterative Processes, Research Notes in Mathematics, 103, Pitman, London, 1984.

[6] L. B. Rall, Computational Solution of Nonlinear Operator Equations, Robert E. Krieger Publishing Company, Inc., New York, 1979. 
[7] J. F. Traub, Iterative Methods for the Solutionm of Equations, Prentice Hall., Englewood Cliffs. N. J., 1964.

Universidad de La Rioja, Departamento de Matemáticas y Computacion. C/Luis de Ulloa s/n. 26004, Logrono. Spain.

E-mail: jezquer@dmc.unirioja.es

E-mail: mahernan@dmc.unirioja.es

E-mail: masalano@dmc.unirioja.es 